\title{
BMJ Open Effect of daily application of a $0.05 \%$ chlorhexidine solution on the incidence of (aspiration) pneumonia in care home residents: design of a multicentre cluster randomised controlled clinical trial
}

\author{
Vanessa Hollaar, ${ }^{1,2,3}$ Claar van der Maarel-Wierink, ${ }^{1,4}$ Gert-Jan van der Putten, ${ }^{1,3,5}$ \\ Bert de Swart, ${ }^{2,6}$ Cees de Baat ${ }^{1,3}$
}

To cite: Hollaar V, MaarelWierink $C$ van der, van der Putten G-J, et al. Effect of daily application of a $0.05 \%$ chlorhexidine solution on the incidence of (aspiration) pneumonia in care home residents: design of a multicentre cluster randomised controlled clinical trial. BMJ Open 2015;5: e007889. doi:10.1136/ bmjopen-2015-007889

- Prepublication history for this paper is available online. To view these files please visit the journal online (http://dx.doi.org/10.1136/ bmjopen-2015-007889).

Received 10 February 2015 Revised 15 August 2015 Accepted 18 August 2015

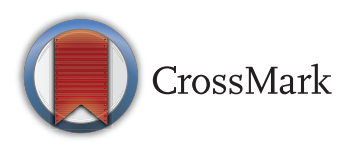

For numbered affiliations see end of article.

Correspondence to Vanessa Hollaar; vanessa.hollaar@ radboudumc.n

\section{ABSTRACT}

Introduction: Pneumonia is an important cause of death in care home residents. Dysphagia and poor oral health are significant risk factors for

developing aspiration pneumonia. Oral hygiene care reduces the number of oral bacteria and the risk of aspiration pneumonia. However, it is not clear yet which oral hygiene care intervention is most efficacious in reducing the risk of aspiration pneumonia. The aim of the study is to assess whether the application of a $0.05 \%$ chlorhexidine-containing solution in addition to the usual daily oral hygiene care reduces the incidence of pneumonia in physically disabled care home residents with dysphagia.

Methods and analysis: The study was designed as a multicentre cluster randomised controlled clinical trial, with care homes as units of randomisation. During 1 year, 500 physically disabled care home residents with dysphagia will be followed. The intervention consists of applying a $0.05 \%$ chlorhexidine-containing solution twice daily, immediately after the usual oral hygiene care, whereas the control group receives no application after the usual oral hygiene care. The primary outcome is the incidence of pneumonia diagnosed by a physician, using a set of strictly described criteria. The effect of the intervention on the incidence of pneumonia will be determined using a Cox regression analysis. The secondary outcomes are correlations between incidence of pneumonia, age, gender, diagnosed diseases, dysphagia severity, care dependency, actually used medication, number of teeth and implants present and the presence of removable dentures.

Ethics and dissemination: Ethical approval was obtained from the Medical Ethical Committee of Radboud university medical centre: NL.nr: 41990.091.12. Written and informed consent will be obtained from all participating care homes and residents. The study's findings will be published in peer-reviewed journals.

\section{Strengths and limitations of this study}

- This is the first multicentre cluster randomised controlled clinical trial which studies the effect of a daily application of a $0.05 \%$ chlorhexidine solution on the incidence of aspiration pneumonia in care home residents.

- The results of this study will contribute to the improvement of oral hygiene care for care home residents.

- A limitation of this study is a potential sample selection bias, because the cluster randomisation may result in random effect and cluster selection bias.

Trial registration number: The trial has been registered in the Netherlands in the National Trial Register: $\mathrm{TC}=3515$.

\section{INTRODUCTION}

In care homes, pneumonia is the second most common infection after urinary tract infection, and the leading cause of death from infection. ${ }^{1}$ The incidence of pneumonia in care homes is 10 -fold the incidence of pneumonia in the community. ${ }^{2}$ The risk of developing aspiration pneumonia in care home residents is also higher than in community-dwelling older people. ${ }^{3}$ Aspiration, with the risk of developing aspiration pneumonia, is defined as the inhalation of oropharyngeal or gastric contents into the larynx and lower respiratory tract. ${ }^{4}$ Dysphagia is involved in developing 
aspiration pneumonia. ${ }^{5}$ A meta-analysis on dysphagia and aspiration pneumonia in frail older people confirmed that dysphagia is a significant risk factor for aspiration pneumonia with frail elderly people $(\mathrm{OR}=9.84$; 95\% CI 4.15 to 23.33 ), particularly with elderly people suffering from cerebrovascular disease: $\mathrm{OR}=12.93 ; 95 \%$ CI 8.61 to $19.44 .{ }^{6}$ Poor oral hygiene care in care homes causes the accumulation of dental plaque and colonisation of oral surfaces and dentures with respiratory pathogens. Aspiration of these pathogens might cause lower respiratory tract infections such as pneumonia. ${ }^{7-10}$ It is not the type of oral bacteria, but the amount of inhaled bacteria which is an important factor in developing aspiration pneumonia. ${ }^{11}$

Care home residents often depend on nurses for their daily oral hygiene care. It has been found that care home residents in several countries have poorly cleaned teeth and removable dentures. ${ }^{12}$ Although it has been suggested that oral hygiene care, such as brushing the teeth after each meal, cleansing dentures once daily, and professional oral healthcare once weekly, reduces the number of oral bacteria in care home residents sufficiently, it is not quite clear which oral healthcare intervention is most efficacious in reducing the risk of aspiration pneumonia. ${ }^{8} 1314$ A systematic review and meta-analysis has found a significant reduction of pneumonia in an intensive care unit among cardiac surgery patients receiving mechanical ventilation and oral hygiene care with different concentrations of chlorhexidine gluconate solutions. No significant reduction was found among non-cardiac surgery patients. ${ }^{15}$ To the best of our knowledge, no data are available on the reduction of pneumonia in care home residents using a chlorhexidine-containing solution additional to their daily oral hygiene care.

Recently, the literature published between January 2000 and April 2009 was systematically reviewed in order to find risk factors of aspiration pneumonia in frail elderly people. ${ }^{16}$ The following risk factors could be identified: age, male gender, lung diseases, dysphagia, diabetes mellitus, severe dementia, ACE I/D genotype deletion/deletion genotype, poor oral health, malnutrition, Parkinson's disease, the use of antipsychotic drugs, and the use of proton-pump inhibitors. It was suggested that the presence of two or more of these risk factors could be an indicator that specific preventive oral healthcare is necessary for attempting to reduce the risk of developing aspiration pneumonia. ${ }^{16}$

The results of the aforementioned studies showed that frail older people have a high risk of dysphagia with, as a consequence, a higher risk of developing aspiration pneumonia than individuals without dysphagia, and that the application of a chlorhexidine-containing solution in addition to usual daily oral hygiene care may reduce the risk of developing aspiration pneumonia. Therefore, the aim of the study is to assess whether the application of a $0.05 \%$ chlorhexidine-containing solution in addition to daily oral hygiene care reduces the incidence of pneumonia in physically disabled care home residents with dysphagia.

\section{STUDY OBJECTIVES}

The objectives of the study are rendered in the following research questions:

1. Is there any statistically significant difference with regard to the incidence of (aspiration) pneumonia between physically disabled care home residents with dysphagia, who in addition to their usual daily oral hygiene care use a $0.05 \%$ chlorhexidine-containing solution (intervention group), and physically disabled care home residents with dysphagia, who only receive the usual daily oral hygiene care (control group)?

2. Is there any statistically significant correlation between age, gender, diagnosed diseases, dysphagia severity, care dependency, actually used medication, the number of teeth and implants present, and the presence of removable dentures and the incidence of pneumonia in physically disabled care home residents with dysphagia who did or did not apply a $0.05 \%$ chlorhexidine-containing solution to their usual oral hygiene care?

\section{METHODS AND ANALYSIS}

\section{Study design and setting}

This study protocol was developed in accordance with the Consolidated Standards of Reporting Trials (CONSORT) guidelines. ${ }^{17}$ The study design is a multicentre cluster randomised controlled clinical trial, with care homes as units of randomisation (figure 1). The study will be performed in different care home units in the Netherlands. After approaching the care homes, the care homes will be allocated randomly to either the intervention group or the control group, assigned by lot. The sample size of the participants in both groups will be monitored. The enrolment of all care home units will take place at different moments within 1 year. However, in one care home unit, all participants will start at the same time.

\section{Power calculation}

On the basis of a power calculation, 500 physically disabled care home residents with dysphagia will be followed during 1 year. The care homes are the units of randomisation and will be allocated randomly to the intervention or control group (figure 1). Factors taken into account in the sample size calculations are the distribution of the outcome variable in the population $(\sigma)$ and the presumed effect of the intervention $\left(\mu^{1}-\mu^{2}\right)$, the requested power $(1-\beta)$, the $\alpha$ and the value of the intraclass correlation (design effect). The primary formula used to calculate the sample size is:

$$
\mathrm{n}=\frac{2 \sigma^{2}}{\left(\mu^{1}-\mu^{2}\right)^{2}} \times \mathrm{f}(\alpha, \beta)
$$

Calculating the power, the incidence of pneumonia in care home residents is, in agreement with not internationally published incidence data for the year 2011, 


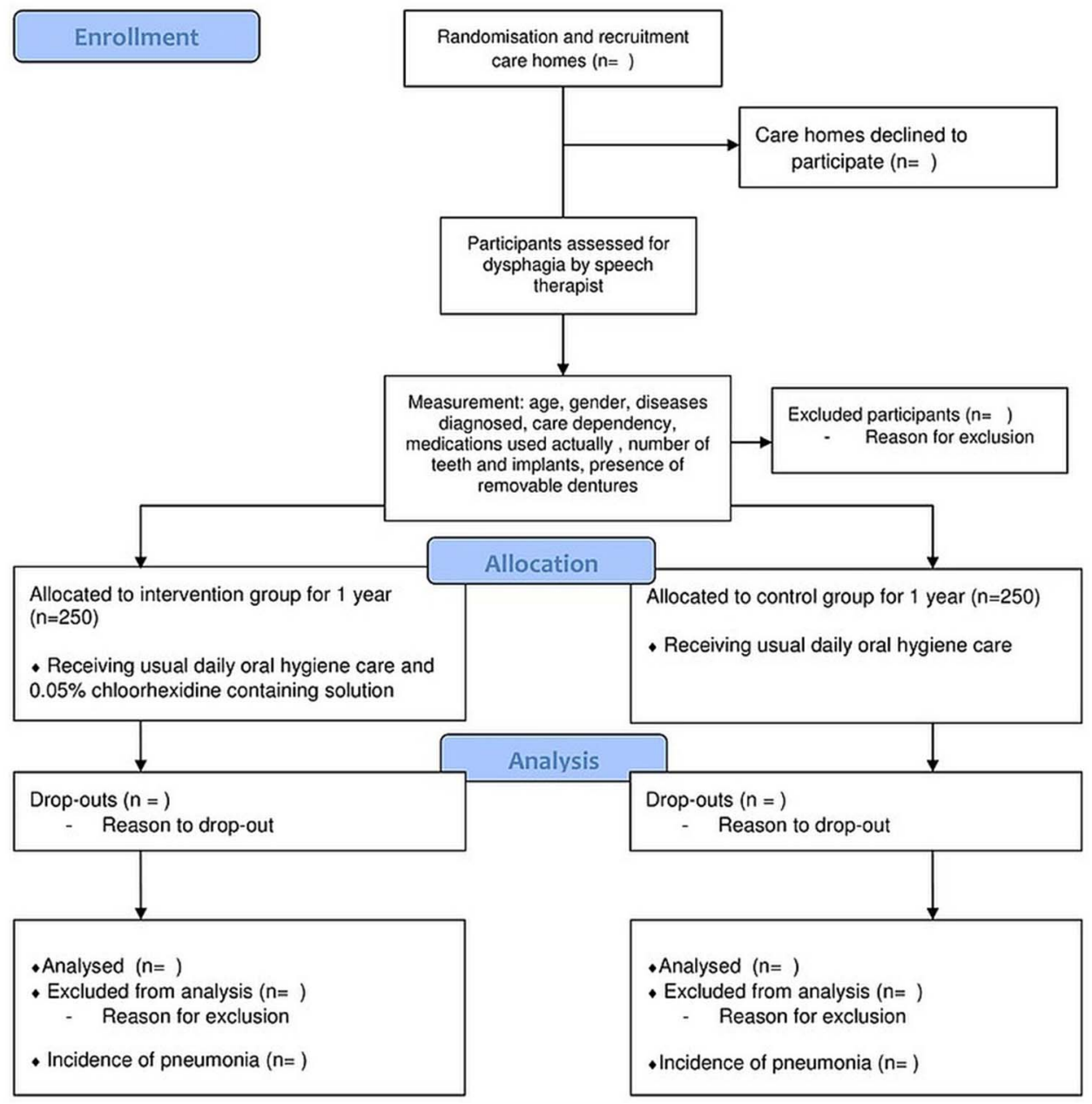

Figure 1 Presents a flow chart of the multicentre cluster randomised controlled clinical trial.

largely assessed as $25 \%,{ }^{18}$ the presumed reduction of the incidence is $40 \%$, the requested power is 0.8 , and the $\alpha$ is 0.05 . If the true relative risk of failure of residents of the intervention group relative to residents of the control group is $0.6,250$ residents are needed in the intervention group and 250 residents in the control group in order to be able to reject the hypothesis that the risk difference equals $0 \%$ with probability (power) 0.8 .

\section{Recruitment of care homes and participants}

The care homes will be selected from a database. Care home units with residents for short-term care or with cognitively impaired residents (mainly suffering from dementia) will be excluded from this study. After recruiting the care homes, the care homes will randomly be allocated to either the intervention group or the control group, assigned by lot. The care homes will receive information on the study in detail by post and email. After this mailing, all care homes will be called. If the care home agrees to participate, the principal investigator $(\mathrm{VH})$ will organise an information meeting. Written informed consent by the board of the care home will be received before the start of the study. After this informed consent, recruitment of residents will take place. Only physically disabled residents with dysphagia are eligible to participate.

In the Netherlands, care homes contain somatic and psychogeriatric wards of primarily physically disabled residents and/or primarily cognitively impaired residents, respectively. The most common medical diagnoses in primarily physically disabled residents are cerebrovascular accidents (26\%), other neurological diseases $(7 \%)$, post-hip surgery status and other locomotor disorders $(30 \%)$, malignancies $(8 \%)$ and other miscellaneous diagnoses (29\%). ${ }^{19}$ More than $85 \%$ of residents of psychogeriatric wards suffer from dementia. Most residents show a wide range of disabilities and handicaps, affecting autonomous activities of daily living, personal care, mobility and mental functions. The nursing staff, speech therapist and the responsible elderly care physician ${ }^{20}$ indicate if the resident on a somatic ward is $\operatorname{cog}$ nitively and physically able to participate and is able to conduct the given instructions by the nursing staff.

Participants are recruited by a physician and a speech therapist of the care home. The recruited residents or their legal representatives will receive information, both by word of mouth and written, about the study. An information letter and the informed consent letter will be 
presented by the principal investigator or the nursing staff. Before giving their written informed consent, participants and/or their legal representatives will be given the opportunity to ask additional questions about the study.

\section{Inclusion}

Residents will be included when they meet the following criteria: aged 65 years or older, physically disabled and diagnosed with dysphagia. Dysphagia is defined as: "Difficulty with any stage of swallowing (oral, pharyngeal, oesophageal) and dysfunction in any stage or process of oral intake; includes any difficulty in the passage of food, liquid, or medicine during any stage of swallowing that impairs the client's ability to swallow independently or safely". ${ }^{21}$ In this study, dysphagia will be determined as a dysfunction in the process of oral intake using the Functional Oral Intake Scale (FOIS).$^{22}{ }^{23}$ The FOIS reflects the functional oral intake of patients with dysphagia and has adequate reliability, validity and sensitivity to change in functional oral intake in patients with stroke. ${ }^{23}$ It is a seven-point scale (level 1-7), developed to systematically rate the functional level of oral intake of food and liquid. Residents with level 1 (nothing by mouth) till level 6 (total oral diet with multiple consistencies without special preparation but with specific food limitations) will be included. Level 7 shows a total oral diet with no restrictions and therefore no dysphagia. Dysphagia will be diagnosed by a speech therapist.

Residents who are cognitively impaired (mainly suffering from dementia), in a coma or vegetative state, terminally ill, dependent on mechanical ventilation, in day care, in short-term care, or already using an oral hygiene care solution, will be excluded. A physician has to withdraw a resident from the study when the resident's conditions have altered according to one of the exclusion criteria. However, in case pneumonia is the reason for the condition alteration, the pneumonia will be registered as a case with respect to the outcome variable. Withdrawn residents will not be replaced because otherwise, in order to determine the incidence of pneumonia, significant bias would be introduced by replacement.

\section{Intervention and control groups}

The intervention consists of applying a $0.05 \%$ chlorhexidine-containing solution (Perio-aid Maintenance $0.05 \%$, Dentaid BeNeLux BV) twice daily immediately after the usual oral hygiene care. The application method depends on the severity of the dysphagia. The speech therapist indicates the application method by considering the FOIS level with respect to the toleration of thin liquids by the resident. Residents who tolerate thin liquids have to rinse with the $0.05 \%$ chlorhexidinecontaining solution for $30 \mathrm{~s}$ twice daily immediately after the usual oral hygiene care. Residents with severe dysphagia, who cannot tolerate thin liquids, have to clean their teeth, gums, tongue, palate, and buccal mucosa with a gauze containing a $0.05 \%$ chlorhexidinecontaining solution twice daily, immediately after the usual oral hygiene care. The control group receives the usual oral hygiene care without addition of an oral rinse. In both groups, the usual oral hygiene care is not standardised. When the usual oral hygiene care would be standardised, this would be an intervention as well and no effect of the additional chlorhexidine-containing solution could be established.

\section{Monitoring}

In the intervention group, the application of the chlorhexidine-containing solution will be guided and monitored after each period of 2 weeks by a group of visiting study assistants who have been instructed by the principal investigator about the use of a strict guiding and monitoring protocol. Every study assistant will visit the same care home ward(s) each time. In each care home, two trained internal study supervisors will monitor the usual daily oral hygiene care, including the application of the chlorhexidine-containing solution. The internal study supervisors will be nurses, dental hygienists or dentists. After every 2 weeks, the study assistants will monitor whether the chlorhexidine-containing solution has been used. The study assistants will monitor the potential side effects of the chlorhexidinecontaining solution by oral assessments and by evaluations with the internal study supervisors. After each visit, the study assistants will report their findings using a standardised Clinical Report Form (CRF). The principal investigator will visit the intervention groups to evaluate the study with the internal study supervisors after 6 and 12 months after the start of the study. During those visits, the principal investigator will also do oral assessments with the participants in the intervention group for potential side effects of the chlorhexidine-containing solution. On top of that, the control groups will be monitored with the same monitoring protocol after each period of 4 weeks. In the control groups, the principal investigator will evaluate the study with the internal study supervisors at 6 months and at the end of the study. No follow-up monitoring of the participated care home residents will take place after the end of the study.

\section{Data collection}

Research data will be gathered at baseline by the principal investigator (the first author of this article) and the assistant examiners (dental hygienists and bachelor dental hygiene students). The assistant examiners are educated by the principal investigator to perform a short standardised oral examination and to gather data from the residents' medical records. This short standardised oral examination will determine the number of teeth and implants present and the presence of removable dentures. The following additional data are gathered from the patient's medical record: age, gender, diagnosed diseases and actually used medication. An 
overview of the data which will be collected is presented in table 1. Subsequently, the level of care dependency will be determined by a care provider using the Care Dependency Scale. ${ }^{25}$

In case symptoms of pneumonia occur in the participating residents during this study, they will be physically examined by a physician. If pneumonia is diagnosed, the physician will inform the principal investigator in order to register the pneumonia as a case with respect to the outcome variable. The diagnosis and related clinical symptoms of pneumonia will be registered using a CRF. When a participant has more than one episode of pneumonia during the trial, all episodes will be monitored and reported. Any other alterations in medical conditions of the participant, such as hospitalisation, death or discontinuation of the study, will be reported by the physician using different CRFs. All CRFs will be collected by the assistant examiners every 2 weeks.

\section{Outcome measures}

The primary outcome variable is the incidence of pneumonia diagnosed by a physician, using a set of strictly described criteria. Since uniform criteria for diagnosing pneumonia in care home residents are lacking, a set of criteria will be assessed by a Delphi panel of experts before the start of the study. To determine the incidence of pneumonia, every episode of pneumonia with the included residents within the study period of 1 year will be registered.

Secondary outcomes are correlations between age, gender, diagnosed diseases, dysphagia severity, care dependency, actually used medication, number of teeth and implants present, and presence of removable dentures and the incidence of pneumonia in physically disabled care home residents with dysphagia who did or did not apply a $0.05 \%$ chlorhexidine-containing solution to their usual oral hygiene care.

\section{Statistical analysis}

The categorical and continuous variables will initially be subjected to an exploratory data analysis, applying a variety of mostly graphical methods and methods for testing the necessary assumptions. The effect of the intervention on the incidence of pneumonia will be determined using a Cox regression analysis. The intervention will be the independent variable, whereas the dependent variable is the occurrence of pneumonia. In addition to this main analysis, the influence of the other research variables on the incidence of pneumonia will be determined using an elaborate Cox regression analysis (age, gender, diseases diagnosed, dysphagia severity, care dependency, actually used medication, number of teeth and implants present and presence of removable dentures). These variables can be used in case any imbalance emerges in the clustering of the care homes. In this survival model, a frailty model which aims to account for heterogeneity caused by unmeasured covariates will be included to take into account the clustering of data within care homes. A 95\% CI will be used with all estimated values. Recording the moment and the reason of withdrawal of participating residents facilitates the use of the data gathered from all participating residents for statistical analysis, even if they were withdrawn prematurely.

\section{ETHICS AND DISSEMINATION}

The study is conducted according to the principles of the Declaration of Helsinki (V.7c, 2004) and in accordance with the Medical Research Involving Human

Table 1 Data collected, outcomes, scales and collection moment

\begin{tabular}{lll}
\hline Data & Outcomes/scales & Collection moment \\
\hline Functional Oral Intake & Level 1: Nothing by mouth & Baseline \\
Scale & Level 2: Tube dependent with minimal attempts of food or liquid & \\
& Level 3: Tube dependent with consistent oral intake of food or liquid & \\
& Level 4: Total oral diet of a single consistency & Level 5: Total oral diet with multiple consistencies, but requiring special \\
& preparation or compensations & \\
& Level 6: Total oral diet with multiple consistencies without special & Baseline \\
& preparation, but with specific food limitations & \\
Level 7: Total oral diet with no restrictions & \\
Scale & Completely care dependent, score <25 & Baseline \\
& To a great extent care dependent, score 25-44 & Baseline \\
Patient's medical & Partially care dependent, score 45-59 & When symptoms occur \\
record & Almost independent, score $>69$ & during study \\
Oral examination & Mender, age & \\
Pneumonia & Number of teeth and implants, presence of removable dentures & \\
\hline
\end{tabular}


Subjects ACT (WMO). The trial has been registered in the Netherlands in the National Trial Register: $\mathrm{TC}=3515$.

Written and informed consent will be contained from all participating care homes and care home residents. In case a resident is incapacitated, their legal representative will be asked to consent instead. Regardless of informed consent, signs of objection expressed by the resident or deterioration of their health will be closely monitored. If any objection or deterioration of their health occurs, the study will be stopped immediately. Possible adverse events related to study participation will also be monitored closely. Implementation and conduct of the study will be monitored by the investigators (authors).

CRF will be handled and stored confidentially. These CRFs and residents' personal data will be stored in two different files in order to ensure confidentiality. The data from both files cannot be traced to the participating residents. To safeguard these files, only the investigators have access to them by using a password.

The results of this study will be published in peerreviewed journals and in relevant news channels for clinicians. Duplicate publication will be avoided.

\section{DISCUSSION}

Using cluster randomisation may result in random effect and cluster selection bias. Therefore, a frailty model which aims to account for heterogeneity, caused by unmeasured covariates, will be included in the Cox regression analysis to take into account the clustering of data within care homes.

Chlorhexidine is a widely used oral rinse that is often applied in critically ill populations. ${ }^{26}{ }^{27}$ Adverse effects due to chlorhexidine are rare, but have been reported. Very rarely, applying a chlorhexidine-containing solution causes an allergic and/or hypersensitivity reaction. Other side effects are discoloration of teeth, removable dentures, mucosa or tongue, temporary taste disorder, swelling of salivary glands, and pain. However, these side effects may present themselves when using a concentration of $0.2 \%$, but very rarely when using a lower concentration, whereas the concentration used in this study is $0.05 \%$. Oral ingestion of chlorhexidine is usually well tolerated because of the negligible systemic absorption. ${ }^{10}$

Although it is not possible to comment on the effectiveness of the intervention in advance, the benefits for the residents in the intervention group may be a better oral hygiene status and oral health condition and, consequently, a reduction in the risk of developing pneumonia. The favourable results of studies about using chlorhexidine to prevent pneumonia with patients who depend on mechanical ventilation are promising for a similar result in preventing aspiration pneumonia in physically disabled care home residents with dysphagia. ${ }^{28}$ The literature shows that structured, adequate oral healthcare is necessary for the maintenance and improvement in the quality of life of elderly people. ${ }^{29} 30$ Preventing aspiration pneumonia by improving oral healthcare may be a significant contribution to the quality of life of care home residents.

\section{Author affiliations}

${ }^{1}$ BENECOMO, Flemish-Netherlands Geriatric Oral Research Group, Nijmegen, The Netherlands

${ }^{2}$ Department of Neurorehabilitation, HAN University of Applied Sciences, Nijmegen, The Netherlands

${ }^{3}$ Department of Oral Function and Prosthetic Dentistry, Radboud University Medical Centre, Nijmegen, The Netherlands

${ }^{4}$ Center for Special Care in Dentistry, Amsterdam, The Netherlands

${ }^{5}$ Amaris Gooizicht, Hilversum, The Netherlands

${ }^{6}$ Division Speech Therapy, Department of Rehabilitation, Radboud University Medical Centre, Nijmegen, The Netherlands

\section{Twitter Follow Vanessa Hollaar at @oralevidence}

Contributors CvdM-W developed the first draft of this study. VH, CvdM-W, G-JvdP, BdS and CdB contributed to the further development and design of the study protocol. All the authors critically reviewed several drafts and approved the final manuscript. VH is the principal investigator and will coach the team of assistant examiners to collect the research data. G-JvdP will support VH in setting up the trial; he will assist with the logistic aspects of the trial and is responsible for the statistical analysis of the collected research data.

Funding Dentaid BeNeLux BV will provide free shipping of the oral rinse (Perio-aid Maintenance $0.05 \%$ ) to the participating care homes.

\section{Competing interests None declared.}

Ethics approval Medical Ethical Committee of Radboud university medical centre has approved the study (NL.nr:41990.091.12).

Provenance and peer review Not commissioned; externally peer reviewed.

Open Access This is an Open Access article distributed in accordance with the Creative Commons Attribution Non Commercial (CC BY-NC 4.0) license, which permits others to distribute, remix, adapt, build upon this work noncommercially, and license their derivative works on different terms, provided the original work is properly cited and the use is non-commercial. See: http:// creativecommons.org/licenses/by-nc/4.0/

\section{REFERENCES}

1. Pace $\mathrm{CC}, \mathrm{McC}$ cullough $\mathrm{GH}$. The association between oral microorganisms and aspiration pneumonia in the institutionalized elderly: review and recommendations. Dysphagia 2010;25:307-22.

2. Marrie TJ. Pneumonia in the long-term-care facility. Infect Control Hosp Epidemiol 2002:23:159-64.

3. Reza Shariatzadeh M, Huang JQ, Marrie TJ. Differences in the features of aspiration pneumonia according to site of acquisition: community or continuing care facility. J Am Geriatr Soc 2006;54:296-302.

4. Marik PE. Aspiration pneumonitis and aspiration pneumonia. N Engl J Med 2001;344:665-71.

5. Marik PE, Kaplan D. Aspiration pneumonia and dysphagia in the elderly. Chest 2003;124:328-36.

6. van der Maarel-Wierink CD, Vanobbergen JNO, Bronkhorst EM, et al. Meta-analysis of dysphagia and aspiration pneumonia in frail elders. J Dent Res 2011;90:1398-404.

7. Scannapieco FA. Role of oral bacteria in respiratory infection. J Periodontol 1999;70:793-802.

8. Yoneyama T, Yoshida M, Ohrui T, et al., Oral Care Working Group. Oral care reduces pneumonia in older patients in nursing homes. J Am Geriatr Soc 2002;50:430-3.

9. Quagliarello V, Ginter S, Han L, et al. Modifiable risk factors for nursing home-acquired pneumonia. Clin Infect Dis 2005;40:1-6.

10. El-Solh AA. Association between pneumonia and oral care in nursing home residents. Lung 2011;189:173-80.

11. Ingles TJ, Sherratt MJ, Sproat LJ, et al. Gastroduodenal dysfunction and bacterial colonization of the ventilated lung. Lancet 1993;341:911-13.

12. De Visschere LM, Grooten L, Theuniers G, et al. Oral hygiene of elderly people in long-term care institutions-a cross-sectional study Gerodontology 2006;23:195-204.

13. Bassim CW, Gibson G, Ward T, et al. Modification of the risk of mortality from pneumonia with oral hygiene care. J Am Geriatr Soc 2008;56:1601-7. 
14. Ishikawa A, Yoneyama T, Hirota K, et al. Professional oral health care reduces the number of oropharyngeal bacteria. J Dent Res 2008;87:594-8.

15. Klompas M, Speck K, Howell MD, et al. Reappraisal of routine ora care with chlorhexidine gluconate for patients receiving mechanical ventilation: systematic review and meta-analysis. JAMA Intern Med 2014; 174:751-61.

16. van der Maarel-Wierink CD, Vanobbergen JNO, Bronkhorst EM, et al. Risk factors for aspiration pneumonia in frail older people: a systematic literature review. J Am Med Dir Assoc 2011;12:344-54.

17. Schulz KF, Altman DG, Moher D, et al. CONSORT 2010 Statement: updated guidelines for reporting parallel group randomised trials. BMC Med 2010;8:18.

18. Kerpiçlik F, Haenen A, Alblas J, et al. Surveillance Netwerk Infectieziekten Verpleeghuizen (SNIV). Referentiecijfers basis surveillance 2011. Bilthoven: Rijksinstituut voor Volksgezondheid en Milieu, 2012.

19. Schols JM. Nursing home medicine in The Netherlands. Eur J Gen Pract 2005;11:141-3.

20. Koopmans RT, Lavrijsen JC, Hoek JF, et al. Dutch elderly care physician: a new generation of nursing home physician specialists. $J$ Am Geriatr Soc 2010;58:1807-9.

21. Clark GF, Avery-Smith W, Wold LS, et al. Specialized knowledge and skills in feeding, eating, and swallowing for occupational therapy practice. Am J Occup Ther 2007;61:686-700.
22. Hansen T, Kjaersgaard A, Faber J. Measuring elderly dysphagic patients' performance in eating-a review. Disabil Rehabil 2011;33:1931-40

23. Crary MA, Carnaby Mann GD, Groher ME. Initial psychometric assessment of a Functional Oral Intake Scale for dysphagia in stroke patients. Arch Phys Med Rehabil 2005;86:1516-20.

24. McMicken BL, Muzzy CL, Calahan S. Retrospective ratings of 100 first time-documented stroke patients on the Functional Oral Intake Scale. Disabil Rehabil 2010;32:1163-72.

25. Dijkstra A, Tiesinga J, Plantinga L, et al. Diagnostic accuracy of the care dependency scale. J Adv Nurs 2005;50:410-16.

26. O'Reilly M. Oral care of the critically ill: a review of the literature and guidelines for practice. Aust Crit Care 2003;16:101-10.

27. Berry AM, Davidson PM, Masters J, et al. Systematic literature review of oral hygiene practices for intensive care patients receiving mechanical ventilation. Am J Crit Care 2007;16:552-62.

28. Beraldo CC, Andrade D. Oral hygiene with chlorhexidine in preventing pneumonia associated with mechanical ventilation. J Bras Pneumol 2008;34:707-14.

29. Ghezzi EM, Ship JA. Systemic diseases and their treatments in the elderly: impact on oral health. J Public Health Dent 2000;60: 289-96.

30. Kandelman D, Petersen PE, Ueda H. Oral health, general health and quality of life in older people. Spec Care Dentist 2008;28:224-36. 